Silvana Maria Orestes-Cardoso

Alan Bruno Lira de Farias ${ }^{2}$

Marianna Ribeiro Medeiros Guerra Pereira $^{3}$

Antonio Jorge Orestes-Cardoso ${ }^{4}$

Irani de Farias Cunha Júnior ${ }^{5}$

\section{Acidentes perfurocortantes: prevalência e medidas profiláticas em alunos de odontologia*}

\author{
Needlestick and sharp injuries - prevalence and prophylatic \\ measures among dentistry students
}

\begin{abstract}
${ }^{1}$ Doutora pela Universidade de Paris VII (França), Professora do Departamento de Prótese e Cirurgia Buco-Facial da Universidade Federal de Pernambuco (UFPE), Recife, PE.

${ }^{2}$ Cirurgião-dentista. Mestrando pela Universidade Federal de Pernambuco (UFPE), Recife, PE.
\end{abstract}

${ }^{3}$ Cirurgiã-dentista pela Universidade Federal de Pernambuco (UFPE), Recife, PE.

${ }^{4}$ Mestre pela Faculdade de Odontologia de Pernambuco (FOP-UPE). Professor da Faculdade de Odontologia de Pernambuco, Recife, PE.

${ }^{5}$ Mestre pela UFPE, Professor do Departamento de Prótese e Cirurgia Buco-Facial da Universidade Federal de Pernambuco (UFPE), Recife, PE.

Contato:

Prof $^{\mathrm{a}}$. Dr ${ }^{\mathrm{a}}$. Silvana Maria OrestesCardoso

Rua Djalma Farias, 29, Torreão - Recife - Pernambuco - Brasil.

CEP: 52030-190

E-mail:

silvanaorestes@hotmail.com

*Este trabalho foi subsidiado pelo Conselho Nacional de Desenvolvimento Científico e Tecnológico (CNPq), através do Programa Institucional de Bolsas de Iniciação Científica - PIBIC.
Recebido: 27/08/2007

Revisado: 09/04/2009

Aprovado: 13/04/2009

\section{Resumo}

Doenças infecto-contagiosas têm estimulado pesquisas sobre acidentes ocupacionais e biossegurança entre profissionais da saúde de diferentes países. No Brasil, poucos são os estudos realizados na Odontologia. Este estudo objetivou identificar a prevalência de acidentes perfurocortantes e as medidas profiláticas pós-acidentes em duas Faculdades de Odontologia de Recife, Pernambuco, Brasil. A amostra se constituiu de 300 alunos do ciclo profissional, escolhidos aleatoriamente e entrevistados por um dos pesquisadores. Constatou-se que a prevalência de alunos acidentados nas duas instituições foi de 25,3\%, com percentual mais elevado nos alunos do $8^{\circ}$ ao $10^{\circ}$ períodos (35,3\%). Dos acidentados, $34,2 \%$ foram orientados por professores em relação às medidas profiláticas. A maioria $(73,7 \%)$ se restringiu a lavar o ferimento com água e sabão. Apenas $13,2 \%$ procuraram serviço médico especializado em acidentes ocupacionais, no entanto, 88,7\% classificaram o conhecimento que tinham acerca de medidas profiláticas pós-acidentes de razoável a bom. Os autores concluíram que um percentual ainda elevado de acadêmicos foi submetido à experiência de acidentes perfurocortantes, com potencial de contaminação biológica, fazendo-se necessário potencializar medidas profiláticas no intuito de minimizar tais circunstâncias deletérias à saúde daqueles envolvidos na assistência odontológica.

Palavras-chave: estudantes de odontologia, biossegurança, acidentes ocupacionais.

\begin{abstract}
Studies about infectious-contagious diseases have stimulated the development of research on occupational injuries and biosafety among health care workers in different countries. In Brazil, there are few studies highlighting this topic in Dentistry. This study aimed at identifying the prevalence of needlestick and sharp injuries, and the post-exposure prophylactic measures in two Dentistry schools in Recife, state of Pernambuco, in Brazil. The sample was composed of 300 students randomly chosen at the professional stage of the course, who were interviewed by one of the researchers. The prevalence of the students who had had accidents was $25.3 \%$. Among these, $34.2 \%$ informed they were oriented by teachers to take prophylactic measures. The majority (73.7\%) affirmed having only washed the wound with soap and water. Only $13.2 \%$ sought medical service specialized in occupational injuries. However, $88.7 \%$ classified their knowledge on postexposure prophylactic measures as regular or good. The authors concluded that still a high percentage of students experienced sharp and needlestick injuries, with potential risk of biological contamination. It is, therefore, necessary to enhance the compliance to prophylactic measures in order to minimize such circumstances, which are harmful to the health of those involved in Dentistry Care.
\end{abstract}

Keywords: students, dental, biosafety, occupational injuries. 


\section{Introdução}

A saúde dos trabalhadores é um campo da saúde pública que atua através de procedimentos próprios, visando a promover e proteger a saúde de pessoas no exercício do trabalho. Para tanto é necessária atuação multidisciplinar e interdisciplinar, junto a profissionais especializados, buscando preservação e promoção de saúde através de medidas de alcance coletivo. Os trabalhadores da saúde estão expostos a um processo gerador de doenças profissionais, as quais englobam variados riscos e fatores predisponentes ao desequilíbrio biopsicossocial. É importante salientar que esses riscos e fatores muitas vezes não são encarados com a seriedade que deveriam, gerando agravos à saúde.

A prática da Odontologia, por abranger uma grande variedade de procedimentos com diferentes níveis de complexidade, geralmente implica em contato com secreções da cavidade oral, a exemplo de saliva, sangue e outros tipos de secreções, como as das vias aéreas superiores, além de aerossóis, sendo fator de risco para a transmissão de infecções entre profissionais e pacientes (KONKEWICZ, 2005).

Para fins de conceituação, a exposição a fluidos corporais fica caracterizada pelo contato com membranas mucosas ou pele não intacta, pelo contato com pele intacta quando há envolvimento de áreas extensas por um longo período de tempo, ou por uma injúria percutânea causada por uma agulha contaminada ou algum objeto pontiagudo (MARINO et al., 2001).

$\mathrm{O}$ atendimento de pacientes infectados e a grande prevalência de doenças de repercussão sistêmica, como hepatites, Aids (Síndrome da Imunodeficiência Adquirida), tuberculose e sífilis, entre outras, têm aumentado a conscientização e motivado a classe odontológica a buscar mais informações na tentativa de minimizar as chances de contaminação cruzada. Sangue, qualquer fluido orgânico contendo sangue visível, secreções vaginais e sêmen são materiais biológicos envolvidos na transmissão do HIV (Vírus da Imunodeficiência Humana). Suor, lágrimas, fezes, urina e saliva (não acompanhada de sangue), por sua vez, não representam risco e não há recomendação de quimioprofilaxia e monitoramento sérico para esses casos (MARINO et al., 2001).

Antes da disseminação do HIV, órgãos internacionalmente conhecidos, como a American Dental Association (ADA), já vinham recomendando medidas para controle de infecção nos atendimentos odontológicos. Após a emergência da Aids, iniciou-se um forte movimento para a adoção de um programa efetivo para controle de infecção cruzada nos serviços de saúde como um todo, visando a reduzir esses riscos (CARMO; COSTA, 2001; DISCACCIATI; VILAÇA, 2001; SILVA; PATROCÍNIO; NEVES, 2002).

Tem-se observado, a partir de então, um aumento da atenção ao tema biossegurança, o qual inclui todo procedimento de combate à contaminação, devendo ser, portanto, uma preocupação de todos os serviços relacionados à saúde (CAIXETA; BARBOSA-BRANCO, 2005; GARBIN et al., 2005; GARCIA; ZANETTI-RAMOS, 2004).

No Brasil, a preocupação com os riscos inerentes à atividade profissional do trabalhador fez com que fossem materializadas normas de condutas a serem seguidas pela classe patronal e laboral. Várias determinações dirigidas para profissionais da área da saúde estão contidas na NR-32, aprovada pela Portaria no 485/2005 do Ministério do Trabalho e Emprego (BRASIL, 2005). De acordo com a Portaria $n^{\circ} 3.214 / 1978$, na sua NR-6, do mesmo Ministério (BRASIL, 1978), no caso de agentes insalubres químicos e físicos, equipamentos de proteção individual (EPI), assim como equipamentos de proteção coletiva e controle na fonte, devem ser adotados. Empregado e empregador têm responsabilidade na observância destes equipamentos. Desse modo, constituise em falta a recusa injustificada do uso de equipamentos de segurança.

No caso de agentes biológicos, como em grande parte das situações é impossível ou inviável o controle na fonte ou do ambiente como um todo, a utilização de barreiras de proteção, representadas nesse caso pelos EPI, deve ser conduta prioritária em todas as situações que ofereçam risco (CAIXETA; BARBOSA-BRANCO, 2005).

Estudos sobre acidentes de trabalho com risco potencial de transmissão do HIV em profissionais da saúde têm sido realizados em várias partes do mundo, principalmente nos Estados Unidos. Neste país, RamosGomez et al. (1997) constataram, entre profissionais e estudantes de quatro clínicas de ensino odontológico, uma incidência de 3,53 acidentes a cada 10.000 atendimentos, enquanto no estudo de Younai, Murphy e Kotelchuck (2001) a incidência foi de 2,46/10.000 atendimentos. No Brasil, Martins, Barreto e Rezende (2004) verificaram que $26 \%$ dos dentistas pesquisados sofreram acidentes perfuro-cortantes nos seis meses anteriores à pesquisa e 75\% já haviam sofrido alguma vez durante a vida profissional.

Estudos estimaram ser o risco de transmissão do HIV por exposição percutânea baixo e por exposição da membrana mucosa ainda menor. Quanto às hepatites, o risco de contaminação mostra-se maior que o do HIV, sendo de duas a três vezes mais alto para hepatite B, em comparação com a hepatite C.É importante ressaltar que não existe intervenção específica para prevenir algumas infecções, como a transmissão do vírus da hepatite C após exposição no trabalho. Na existência de vacinas, como no caso da hepatite B, a utilização das mesmas é recomendada para todos os profissionais de saúde (BRANDÃO JUNIOR, 2000; MARTINS; BARRETO, 2003; SCHECHTER; MARANGONI, 1998).

A título de informação, alguns cuidados pós-exposição merecem destaque e foram classificados por etapas, desde o tratamento do sítio de exposição, passando pela notificação, até o controle/monitoramento das con- 
dições dos profissionais de saúde expostos a acidentes (MARINO et al., 2001):

1. Tratamento do sítio de exposição: para exposição cutânea ou percutânea, lavagem com água corrente e soluções antissépticas; para exposições em mucosa, lavar abundantemente com água ou solução fisiológica.

2. Notificação do acidente: Boletim de Acidente de Trabalho;

3. Coleta de amostras de sangue do paciente: realização de testes sorológicos para HIV, hepatite B e C;

4. Coleta de amostras de sangue do profissional: realização de testes sorológicos para HIV, hepatite B e C;

5. Informações adicionais: risco de contaminação e importância de acompanhamento; em caso de materiais contaminados com HIV ou pacientes com sorologia desconhecida, deve-se preservar por 6 meses.

O Ministério da Saúde também preconiza, como medida imediata após acidente envolvendo exposição a material biológico potencialmente contaminado, a lavagem exaustiva do local exposto. Paciente e profissional devem ser submetidos a testes sorológicos para investigar possível infecção prévia por HIV ou HBV, e, caso indicada (paciente-fonte com sorologia positiva ou desconhecida para tais vírus), a quimioprofilaxia deve ser iniciada dentro das primeiras 24 a 48 horas após a exposição (BRASIL, 2006).

Embora a NR-32 estabeleça a obrigatoriedade de comunicação dos acidentes de trabalho, os profissionais freqüentemente não o fazem, pois, muitas vezes, o acidente não gera nenhuma das situações previstas na definição de acidente de trabalho e pode não ter a transmissão caracterizada de imediato ou em curto prazo. A comunicação apenas quando a doença se desenvolve demonstra, claramente, uma negligência no componente preventivo (CAIXETA; BARBOSA-BRANCO, 2005)

Para garantir os benefícios trabalhistas dos empregados em caso de acidente, grande parte dos hospitais procura estabelecer alguma forma de registro desses acidentes, mas, na prática, há falta de normalização desse procedimento e uma deficiente divulgação junto aos profissionais de saúde. Além disso, é necessário o estabelecimento de rotinas relacionadas às medidas de precaução imediatas, bem como as orientações e a disponibilidade da quimioprofilaxia em casos de acidentes com material biológico de pacientes soropositivos para o HIV ou desconhecidos (CAIXETA; BARBOSA-BRANCO, 2005).

Frente ao exposto, o objetivo desse estudo foi verificar a ocorrência de acidentes perfurocortantes entre acadêmicos de Odontologia e as medidas profiláticas imediatas adotadas após exposição a material biológico potencialmente contaminado.

\section{Métodos}

Os dados foram coletados em duas instituições de ensino superior. Uma delas, Faculdade de Odontologia da Universidade Federal de Pernambuco (UFPE), situase na cidade do Recife, Pernambuco. No município de Camaragibe, mesmo estado, está situada a outra instituição pesquisada, a Faculdade de Odontologia da Universidade de Pernambuco (FOP/UPE).

A população do estudo se constituiu de 598 acadêmicos do ciclo profissional (5ํㅡㅁ ao $10^{\circ}$ períodos) da UFPE e FOP-UPE. A amostra foi composta por 25 acadêmicos por período, em cada instituição, escolhidos aleatoriamente, totalizando em 300 os sujeitos da pesquisa.

A coleta de dados foi realizada no período de setembro de 2005 a fevereiro de 2006, sendo aplicado para tanto um questionário elaborado pelos pesquisadores, o qual abordou aspectos como: história de acidentes perfurocortantes durante as atividades do curso, medidas profiláticas imediatas executadas após acidentes desta natureza, caso tenham ocorrido, e o conhecimento de tais medidas por parte do corpo discente.

A aplicação dos questionários foi realizada após a assinatura do Termo de Consentimento Livre e Esclarecido pelos pesquisados, sendo esta assinatura o critério de inclusão na pesquisa.

A tabulação dos dados foi feita por um único pesquisador e, para manter a confiabilidade dos dados, todos os registros foram revistos por mais um dos autores. A análise dos dados foi realizada através de distribuições absolutas e percentuais uni e bivariadas (Técnicas de estatística descritiva) e dos testes Qui-quadrado de Pearson ou Exato de Fisher, quando as condições para utilização do teste Qui-quadrado não foram verificadas.

Os dados foram digitados na planilha Excel e o software utilizado para a obtenção dos cálculos estatísticos foi o Statistical Analysis System (SAS) na versão 8. A margem de erro utilizada na decisão dos testes estatísticos foi de $5,0 \%$

O trabalho foi previamente submetido e aprovado pelo Comitê de Ética em Pesquisa envolvendo Seres Humanos do Centro de Ciências da Saúde da Universidade Federal de Pernambuco (CEP/CCS/UFPE), de acordo com a Resolução no 196/96 do Conselho Nacional de Saúde, com número de protocolo 075/2005.

\section{Resultados}

Através da distribuição dos pesquisados por sexo e faixa etária, foi possível verificar que a maioria tinha de 23 a 25 anos $(64,7 \%)$, seguida pela faixa etária de 19 a 22 anos (22,3\%), havendo predominância do sexo feminino $(70,3 \%)$.

Na Tabela 1 apresentam-se os resultados da avaliação da ocorrência de acidentes com instrumentos 
perfurocortantes, durante o atendimento clínico-ambulatorial ou após (na limpeza de instrumentos). Desta tabela destaca-se que: aproximadamente 1/4 (25,3\%) dos alunos pesquisados já sofreram acidentes com instrumentos perfurocortantes, tendo sido relatados 18,0\% durante e $14,0 \%$ após o atendimento clínico-ambulatorial. A maior freqüência quanto ao número de acidentes sofridos correspondeu à faixa de um a dois acidentes por acadêmico.

$\mathrm{Na}$ avaliação da ocorrência de acidentes de acordo com o período letivo, verificou-se que o percentual de alunos que já sofreu acidentes foi mais elevado entre os que cursavam do $8^{\circ}$ ao $10^{\circ}$ período $(35,3 \%)$. Quando se analisa o número de acidentes durante o atendimento clínico-ambulatorial, observa-se que 90,0\% dos alunos do $5^{\circ}$ ao $7^{\circ}$ períodos e $74 \%$ dos alunos do $8^{\circ}$ ao $10^{\circ}$ períodos não sofreram acidentes durante o atendimento, sendo estes percentuais ainda maiores para acidentes após o atendimento. Diferenças significantes entre os dois subgrupos foram verificadas para cada uma das variáveis analisadas na tabela.

Na Tabela 2 foram analisadas as medidas profiláticas imediatas adotadas pelos 76 alunos que referiram haver sofrido acidentes com instrumentos perfurocortantes. Desta tabela destaca-se que: apenas $34,2 \%$ dos alunos que sofreram acidentes informaram ter procurado o pro- fessor e recebido orientações sobre as medidas profiláticas imediatas; a maioria $(73,7 \%)$ afirmou ter lavado o ferimento com água e sabão; apenas sete no total afirmaram não ter tomado qualquer medida profilática; dez alunos procuraram o serviço médico especializado em casos de acidentes com exposição a material biológico potencialmente contaminado. Outras medidas profiláticas foram tomadas por sete pesquisados, sendo o uso de soluções antissépticas relatado por quatro alunos e a procura do serviço médico para coleta de amostras de sangue relatada por outros três.

A Tabela 3 mostra que, dos 76 acadêmicos acidentados, a maioria $(84,2 \%)$ informou não ter tido dificuldades em procurar o serviço médico especializado no pós-acidente. A freqüência de cada tipo de dificuldade relatada variou de um a três casos, tendo sido mais citadas a demora no atendimento, o desconhecimento do serviço médico e a difícil localização/acesso ao serviço médico.

Os resultados da auto-avaliação do conhecimento dos acadêmicos sobre medidas profiláticas imediatas a serem adotadas no pós-acidente são mostrados na Tabela 4. Pela tabela, verifica-se que os dois maiores percentuais corresponderam aos que auto-avaliaram o conhecimento como razoável (48,7\%) ou bom (40,0\%).

Tabela 1 Ocorrência de acidentes com instrumentos perfurocortantes entre estudantes de odontologia, no atendimento clínico ambulatorial, segundo o período letivo

\begin{tabular}{|c|c|c|c|c|c|c|c|}
\hline \multicolumn{8}{|c|}{ Período } \\
\hline \multirow[t]{2}{*}{ Variáveis } & \multicolumn{2}{|c|}{$5^{0} a 7^{0}$} & \multicolumn{2}{|c|}{$8^{\circ} a 0^{\circ} 0^{\circ}$} & \multicolumn{2}{|c|}{ Grupo total } & \multirow[t]{2}{*}{ Valor de $p$} \\
\hline & $n$ & $\%$ & $n$ & $\%$ & $n$ & $\%$ & \\
\hline \multicolumn{8}{|l|}{ Ocorrência de acidentes } \\
\hline Sim & 23 & 15,3 & 53 & 35,3 & 76 & 25,3 & $\mathrm{p}^{(1)}<0,0001$ \\
\hline Não & 127 & 84,7 & 97 & 64,7 & 224 & 74,7 & \\
\hline Total & 150 & 100,0 & 150 & 100,0 & 300 & 100,0 & \\
\hline \multicolumn{8}{|c|}{ Número de acidentes durante o atendimento } \\
\hline Nenhum & 135 & 90,0 & 111 & 74,0 & 246 & 82,0 & $p^{(2)}=0,0013$ \\
\hline Um a dois & 13 & 8,7 & 32 & 21,3 & 45 & 15,0 & \\
\hline Três ou mais & 1 & 0,7 & 5 & 3,3 & 6 & 2,0 & \\
\hline Não sabe & 1 & 0,7 & 2 & 1,3 & 3 & 1,0 & \\
\hline Total & 150 & 100,0 & 150 & 100,0 & 300 & 100,0 & \\
\hline \multicolumn{8}{|c|}{ Número de acidentes após o atendimento } \\
\hline Nenhum & 138 & 92,0 & 120 & 80,0 & 258 & 86,0 & $p^{(2)}=0,0186$ \\
\hline Um a dois & 9 & 6,0 & 22 & 14,7 & 31 & 10,3 & \\
\hline Três ou mais & 2 & 1,3 & 6 & 4,0 & 8 & 2,7 & \\
\hline Não sabe & 1 & 0,7 & 2 & 1,3 & 3 & 1,0 & \\
\hline Total & 150 & 100,0 & 150 & 100,0 & 300 & 100,0 & \\
\hline
\end{tabular}

(1) - Através do teste Qui-quadrado de Pearson.

(2) - Através do teste Exato de Fisher. 
Tabela 2 Medidas profiláticas imediatas adotadas por 76 alunos de odontologia que referiram haver sofrido acidentes perfurocortantes

\begin{tabular}{lcc}
\hline \multicolumn{1}{c}{ Medidas } & $n$ & $\%$ \\
\hline Procurou o professor(a) e o mesmo(a) o/a orientou & 26 & 34,2 \\
Procurou o serviço médico especializado em acidentes & 10 & 13,2 \\
Lavou o ferimento com água corrente e sabão & 56 & 73,7 \\
Não fez nada & 7 & 9,2 \\
Outros & 7 & 9,2 \\
$\quad \begin{array}{l}\text { Procurou o serviço médico para recolhimento de } \\
\text { amostras de sangue }\end{array}$ & 3 & 3,9 \\
$\quad$ Lavou o ferimento com solução antisséptica & 4 & 5,2 \\
\hline
\end{tabular}

Tabela 3 Existência e tipo de dificuldades na procura por serviço de saúde especializado, no pós-acidente, entre estudantes de odontologia

\begin{tabular}{lcc}
\hline \multicolumn{1}{c}{ Existência e tipos de dificuldades } & $n$ & $\%$ \\
\hline Existência de dificuldades & & \\
$\quad$ Sim & 12 & 15,8 \\
Não & 64 & 84,2 \\
Total & 76 & 100,0 \\
\hline Tipos de dificuldades relatadas & & \\
$\quad$ Colher amostra de sangue do paciente & & \\
$\quad$ Demora no atendimento médico & 1 & 7,7 \\
$\quad$ Desconhecimento do serviço médico & 3 & 23,1 \\
$\quad$ Difícil localização/acesso ao serviço médico & 3 & 23,1 \\
$\quad$ Horário em que ocorreu o acidente dificultou o acesso à & 3 & 23,1 \\
$\quad$ informação e ao atendimento & 2 & 15,4 \\
$\quad$ Todas as possíveis & 1 & 7,7 \\
\hline Total & 13 & 100,0 \\
\hline
\end{tabular}

Tabela 4 Avaliação do auto-conhecimento, por estudantes de odontologia, sobre medidas profiláticas imediatas pós-acidente perfurocortante

\begin{tabular}{|c|c|c|c|}
\hline & Avaliação & $n$ & $\%$ \\
\hline Péssimo & & 6 & 2,0 \\
\hline Ruim & & 20 & 6,7 \\
\hline Razoável & & 146 & 48,7 \\
\hline Bom & & 120 & 40,0 \\
\hline Excelente & & 8 & 2,7 \\
\hline Total & & 300 & 100,0 \\
\hline
\end{tabular}




\section{Discussão}

No presente estudo, a prevalência de acidentes com instrumentos perfurocortantes foi de $25,3 \%$ no total de alunos pesquisados, fato esse preocupante, pois, mesmo com pouco tempo de atividade clínica, de cada quatro alunos, aproximadamente um já esteve exposto a material biológico potencialmente contaminado, o qual pode ocasionar doenças que oferecem risco à vida, como a hepatite (vírus tipo B) e a Síndrome da Imunodeficiência Adquirida (HIV/Aids), entre outras.

Comparativamente, em estudo realizado com estudantes de Odontologia do interior do estado do Paraná, observou-se um percentual de aproximadamente 28,3\% para esse tipo de acidente (RIBEIRO, 2005), valor próximo ao encontrado nesta pesquisa.

Analisando o momento em que esses acidentes ocorreram, pudemos observar que durante o atendimento clínico ambulatorial os alunos estiveram mais suscetíveis ao acidente, com 18,0\% de ocorrência, contra 14\% para acidentes ocorridos após essa atividade.

Acreditamos que esse percentual ainda representa um número elevado de acidentes, levando-se em consideração que esses alunos, tendo recebido informações durante o curso, deveriam conhecer as normas de biossegurança a serem seguidas. Outrossim, a orientação e fiscalização pelos professores deveria mitigar essa ocorrência.

O acometimento em atividades no pós-atendimento faz-nos supor que os alunos, no momento da limpeza/ lavagem do instrumental, sofrem os acidentes por desatenção ou negligência no uso de EPI, possivelmente potencializadas por dificuldade de fiscalização precisa desta etapa por parte de professores ou funcionários. Apesar da usual delegação deste tipo de atividade ao pessoal auxiliar durante a vida profissional, como cirurgiões-dentistas, é importante que a formação profissional os capacite a orientar e supervisionar tais ações.

Estudos sobre exposições a material biológico potencialmente contaminado entre estudantes de Odontologia indicam a falta de experiência clínica como um fator agravante, além das características próprias da profissão que apresentam aspectos facilitadores de acidentes. Revelam ainda que a pouca familiaridade com os procedimentos, o nervosismo, a ansiedade e a supervisão constante do professor, como avaliador, aumentariam o risco de acidentes entre os estudantes (PANAGAKOS; SILVERSTEIN, 1997; RIBEIRO, 2005).

Entre profissionais de saúde, a maioria dos acidentes com contaminação através de material biológico ocorre através de instrumentos de trabalho perfurocortantes, especialmente entre aqueles que pres tam assistência direta aos pacientes e executam procedimentos invasivos, pois empregam predominantemente esse tipo de instrumento na prática diária (BALSAMO; FELLI, 2006).
Os estudos que investigaram infecções ocupacionais nas áreas médica e odontológica indicaram que a exposição repetida aos microrganismos do sangue e de outras secreções resultou em uma incidência mais elevada de determinadas doenças infecciosas nesses profissionais do que a observada na população geral (MOLINARI, 2003).

A utilização inadequada dos equipamentos de proteção individual e a ausência de critérios na manipulação de instrumentos e objetos contaminados na prática odontológica podem ser apontadas como colaboradoras no processo de geração do acidente (BERTI; MOIMAZ; AYRES, 2003).

Independentemente da forma de ensino e da estrutura curricular adotada nas universidades, a prevenção e o controle de infecção devem fazer parte da filosofia da formação dos profissionais da área da saúde, assim como do processo de educação continuada durante o exercício profissional, viabilizando a necessária atualização permanente dos profissionais (TIPPLE et al., 2003).

Com relação às medidas profiláticas imediatas adotadas no pós-acidente, a observação de que apenas $34,2 \%$ dos alunos que sofreram acidentes informaram ter procurado o professor para receber orientações, se traduz como superestima de conhecimento por parte dos mesmos em relação a ocorrências desse tipo, já que a grande maioria $(73,7 \%)$ afirmou ter apenas lavado o ferimento com água e sabão e apenas 10 (13,2\%) procuraram o serviço médico especializado em acidentes com exposição a material biológico potencialmente contaminado. O percentual de alunos que afirmaram não ter tomado qualquer medida profilática $(9,2 \%)$ nos faz crer que estes desconhecem ou ignoram os riscos aos quais estão expostos ou temem as medidas quimioprofiláticas preconizadas. Este tipo de comportamento também pode ter sido favorecido pela avaliação dos acidentes como de pequeno porte ou baixo risco, com volume pequeno de sangue e carga viral reduzida (SCHECHTER; MARANGONI, 1998).

O Ministério da Saúde traz como orientação a ser seguida nas instituições de saúde de todo o país, que acidentes de trabalho com sangue e outros fluidos potencialmente contaminados sejam tratados como casos de emergência médica (BRASIL, 2006). Deve-se lembrar que, apesar de muitas doenças infecciosas serem ocasionadas pela contaminação por meio de acidentes com objetos perfurocortantes, não se pode descartar a contaminação por meio de respingos desses fluidos em mucosas, especialmente a mucosa ocular. Acidentes por esse tipo de exposição ocupam também um dos primeiros lugares em número de ocorrências, apesar de ser menor o risco de contaminação efetiva. Verifica-se, porém, a insuficiência de estudos nessa área.

De acordo com Ministério da Saúde, em caso de sorologia do paciente-fonte positiva ou desconhecida para HIV ou HBV, a quimioprofilaxia pós-exposição ocupacional (PEP) deve ser iniciada o mais rápido possível, 
dentro das primeiras 24 ou 48 horas após o acidente (BRASIL, 2006).

Considerando que as medidas profiláticas pós-exposição não são totalmente eficazes, enfatiza-se a necessidade da divulgação, dentro das universidades, dos protocolos de proteção universal em vigor (BRASIL, 2004), para que os alunos possam exercer com mais segurança suas atividades práticas, reduzindo os riscos de infecção e acidentes durante a prática clínico-ambulatorial. Para tanto, os protocolos devem ser governados por sua aplicação prática e não apenas por considerações legais envolvidas, aumentando a aceitação entre os estudantes e profissionais. Para difundir tal aceitação, recomenda-se informação e treinamento na gerência de riscos, participação de estudantes e profissionais de saúde no desenvolvimento dos protocolos e a sustentação da gerência pelos órgãos envolvidos, nesse caso as próprias universidades, para evitar a reversão ao comportamento habitual precedente (VAN GEMERT-PIJNEN et al., 2006).

Com relação à procura por serviços médicos especializados no pós-acidente, dos acadêmicos acidentados que procuraram esse serviço, a maioria $(84,2 \%)$ informou não ter tido dificuldades nessa procura. Entretanto, alguns estudantes, mesmo que em baixos percentuais, apontam algumas dificuldades, como demora no atendimento, desconhecimento do serviço médico e difícil localização/acesso ao serviço médico.

Osório, Machado e Minayo-Gomez (2005) propuseram um método de análise coletiva de acidentes de trabalho em ambiente hospitalar, que consiste em levar o trabalhador a recriar a situação do acidente, deslocando-se para a posição de observador de seu próprio trabalho. Na primeira etapa da análise, o trabalhador é convidado a mostrar ao analista do trabalho como se deu o acidente; na segunda, a dupla acidentado/analista registra, num diagrama, a sucessão de eventos descrita; na terceira, o registro feito é rediscutido e complementado; na quarta, são avaliadas e executadas, sempre pela dupla acidentado/analista, ações destinadas a prevenir a reincidência do acidente analisado. Neste tipo de análise, parece-nos muito interessante que o acidentado seja levado a refletir sobre a forma de como se deu o fato ocorrido, para que o mesmo possa entender os riscos a que esteve e ainda está exposto e possa proceder de forma coerente na prevenção de acidentes, gerando informação, de forma sistemática, para a ação no campo da vigilância.

Em nível institucional, como nas universidades, a reflexão dos casos ocorridos deve levar ao estabelecimento de um programa efetivo de prevenção e controle de acidentes ocupacionais, observando as principais necessidades de cada instituição.

O percentual considerável de alunos que auto-avaliaram seu conhecimento sobre medidas profiláticas imediatas pós-acidente como razoável ou bom $(48,7 \%$ e 40,0\%, respectivamente) demonstra, mais uma vez, a superestima do conhecimento das atitudes frente a es- ses acidentes, já que poucos tomaram as medidas profiláticas corretas quando da ocorrência dos mesmos.

Enfatizamos, então, a necessidade de se implementar ações educativas permanentes sobre as Medidas de Precauções Universais (MPU's), atualmente denominadas precauções-padrão, e a conscientização da necessidade de empregá-las adequadamente. As precauçõespadrão são formas de prevenção a serem utilizadas na assistência a todos os pacientes, na manipulação de sangue, secreções e excreções e contato com mucosas e pele não-íntegra. Tais medidas incluem a utilização de equipamentos de proteção individual e os cuidados específicos recomendados para manipulação e descarte de materiais contaminados por material orgânico (ALMEIDA; PAGLIUCA; LEITE, 2005). Essas medidas devem ser utilizadas na assistência a todos os pacientes, independentemente do diagnóstico definido ou presumido de doença infecciosa (BRASIL, 2006).

Os acidentes de trabalho, fontes importantes de contaminação, precisam ser prevenidos e para isso faz-se necessário, entre outras medidas, minimizar as falhas humanas. A prevenção dessas falhas se dá exatamente pelo devido treinamento da equipe, demonstração do funcionamento de aparelhos e equipamentos novos, seleção e reciclagem adequada dos funcionários para as diversas funções, informações completas sobre como executar determinadas tarefas, realizações de reuniões periódicas com os funcionários, checagem da compreensão da informação transmitida, acompanhamento de funcionários novos, supervisão dos funcionários, fixação de cartazes com orientações necessárias, entre outros (ALMEIDA; PAGLIUCA; LEITE, 2005).

A avaliação da ocorrência de acidentes segundo o período cursado, mostrou-nos que alunos de períodos mais elevados, correspondentes aos 3 últimos períodos ( $8^{\circ}, 9^{\circ}$ e $10^{\circ}$ ), experimentaram um número mais elevado de acidentes do que aqueles de períodos antecessores (5oㅡ $6^{\circ}$ e $7^{\circ}$ ). Esse fato, por si só, não quer dizer que o primeiro grupo de alunos tenha sido mais desatento ou despreparado para evitar acidentes, mas compreendemos que, devido ao número maior de disciplinas clínicas que esses alunos freqüentam ou já freqüentaram, os mesmos apresentam mais tempo de prática clínica, aumentando, assim, o risco percentual de ocorrência de acidentes entre essa faixa de alunos.

Exposição acidental a material biológico contaminado é sentida como uma situação de risco real de contaminação, assim, é compreensível que o dentista que tenha sofrido as apreensões e angústias relacionadas a um acidente perfurocortante declare menor disposição para o atendimento de pacientes portadores do HIV/Aids (SENNA; GUIMARÃES; PORDEUS, 2005). Esse achado torna-se bastante relevante como núcleo de análise neste estudo, pois considerando as características da prática odontológica, que lida com o uso rotineiro de instrumentos perfurocortantes num campo de visualização restrito, aumentando o risco de lesões percutâneas, acidentes ocorridos durante o período 
acadêmico podem acabar gerando nos profissionais uma menor tolerância e disponibilidade para o atendimento de pacientes sabidamente portadores de doenças infectocontagiosas de maior gravidade e repercussão sistêmica.

Outro agravante é que não foi relatado conhecimento dos alunos quanto à existência de protocolos a serem seguidos para esses casos nas instituições. Esses resultados merecem reflexão mais profunda, tanto das instituições formadoras, quanto do próprio corpo discente, apontando para a necessidade de uma reavaliação da estrutura e dos conteúdos dos treinamentos em serviços oferecidos pelos cursos de Odontologia das instituições pesquisadas, com o intuito de procurar diminuir cada vez mais esse tipo de ocorrência, que acarreta dificuldades à vida pessoal e futuro profissional do estudante acidentado.

Reitera-se, então, que a prevenção de acidentes desse tipo deve ser tida como uma das principais metas a serem atingidas pelas instituições, professores e estudantes, no desempenho das atividades clínicas.

Dessa forma, este estudo sugere que, constatado o risco de exposição a material biológico inerente à catego-

\section{Referências}

ALMEIDA, C. B.; PAGLIUCA, L. M. F.; LEITE, A. L. A. S. Acidentes de trabalho envolvendo os olhos: avaliação de riscos ocupacionais com trabalhadores de enfermagem. Revista Latino-Americana de Enfermagem, Ribeirão Preto, v. 13, n. 5, p. 708-716, out. 2005.

BALSAMO, A. C.; FELLI, V. E. A. Estudo sobre os acidentes de trabalho com exposição aos líquidos corporais humanos em trabalhadores da saúde de um hospital universitário. Revista Latino-Americana de Enfermagem, Ribeirão Preto, v. 14, n. 3, p. 346-353, jun. 2006.

BERTI, M.; MOIMAZ, S. A. S.; AYRES, J. P. S. Métodos de controle de infecção cruzada: uma avaliação do emprego na prática odontológica. Revista Paulista de Odontologia, São Paulo, v. 25, n. 5, p. 30-33, 2003.

BRANDÃO JUNIOR, P. S. Biossegurança e AIDS: as dimensões psicossociais do acidente com material biológico no trabalho em hospital. 2000. $124 \mathrm{f}$. Dissertação (Mestrado)-Escola Nacional de Saúde Pública, Fundação Oswaldo Cruz, Rio de Janeiro, 2000.

BRASIL. Ministério da Saúde. Secretaria de Ciência, Tecnologia e Insumos Estratégicos. Diretrizes gerais para o trabalho em contenção com material biológico. Brasília: Ministério da Saúde, 2004. (Série A. Normas e Manuais Técnicos).

BRASIL. Ministério da Saúde. Secretaria de Atenção a Saúde. Departamento de Ações Programáticas. Exposição a materiais biológicos. Brasília: Ministério da Saúde, 2006. (Série A. Normas e Manuais Técnicos - ria de alunos estudada, é necessário estabelecer estratégias de intervenções urgentes, com medidas que possam abranger questões de ordem administrativa e organizacional das instituições, assim como aquelas relacionadas à educação continuada com ênfase em qualidade de atendimento e prevenção de acidentes.

\section{Conclusão}

Os resultados apresentados permitem concluir que um percentual ainda elevado $(25,3 \%)$ dos acadêmicos pesquisados foi submetido à experiência de acidentes com potencial de contaminação biológica através de instrumentos perfurocortantes. Muitos deles mostraram-se inexperientes ou mal orientados em relação a que atitude tomar frente a um acidente desse gênero, apesar da possível superestima de conhecimentos por parte dos mesmos. Faz-se necessário potencializar medidas profiláticas no intuito de minimizar tais circunstancias deletérias à saúde daqueles envolvidos na relação paciente, docentes e discentes.
Saúde do Trabalhador, 3. Protocolo de Complexidade Diferenciada).

BRASIL. Ministério do Trabalho e Emprego. Portaria n. 3214, de 8 de junho de 1978. Aprova as Normas Regulamentadoras - NR - do Capítulo V, Título II, da Consolidação das Leis do Trabalho, relativas a Segurança e Medicina do Trabalho. Diário Oficial [da] República Federativa do Brasil, Brasília, DF, 06 jul. 1978.

Portaria n. 485, de 11 de novembro de 2005. Aprova a Norma Regulamentadora n. 32 - Segurança e saúde no trabalho em estabelecimentos de saúde. Diário Oficial [da] República Federativa do Brasil, Brasília, DF, 16 nov. 2005.

CAIXETA, R. B.; BARBOSA-BRANCO, A. Acidente de trabalho, com material biológico, em profissionais de saúde de hospitais públicos do Distrito Federal, Brasil, 2002/2003. Cadernos de Saúde Pública, Rio de Janeiro, v. 21, n. 3, p. 737-746, jun. 2005.

CARMO, M. R. C.; COSTA, A. M. D. D. Procedimentos de biossegurança em odontologia. Jornal Brasileiro de Clínica \& Estética em Odontologia, Curitiba, v. 5, n. 26, 2001.

DISCACCIATI, J. A. C.; VILAÇA, E. L. Atendimento odontológico ao portador de HIV: medo, preconceito e ética profissional. Revista Panamericana de Salud Pública, Washington, v. 9, n. 4, p. 234-239, abr. 2001.

GARBIN, A. J. I. et al. Biosecurity in public and private office. Journal of Applied Oral Science, Bauru, v. 13, n. 2, p. 163-166, Apr./June 2005. 
GARCIA, L. P.; ZANETTI-RAMOS, B.G. Gerenciamento dos resíduos de serviços de saúde: uma questão de biossegurança. Cadernos de Saúde Pública, Rio de janeiro, v. 20, n. 3, p. 744-752, jun. 2004.

KONKEWICZ, L. R. Controle de infecção em odontologia. Disponível em: < http://www.cih.com.br/ controle_de_infec\%C3\%A7\%C3\%A3o_em_odontolo. htm>. Acesso em: 14 mar. 2005.

MARINO, C. G. G. et al. Cut and puncture accidents involving health care workers exposed to biological materials. The Brazilian Journal of Infectious Diseases, Salvador, v. 5, n. 5, p. 235-242, Oct. 2001.

MARTINS, A. M. E. B. L.; BARRETO, S. M. Vacinação contra a hepatite B entre cirurgiões dentistas. Revista de Saúde Pública, São Paulo, v. 37, n. 3, p. 333-338, jun. 2003.

MARTINS, A. M. E. B. L.; BARRETO, S. M.; REZENDE, V. L. S. Acidentes do trabalho com instrumentos perfurocortantes entre cirurgiões-dentistas. Revista Brasileira de Medicina do Trabalho, Belo Horizonte, v. 2, n. 4, p. 267-274, 2004.

MOLINARI, J. A. Infection control: its evolution to the current standard precautions. Journal of the American Dental Association, United States, v. 134, n. 5, p. 569574, May 2003

OSÓRIO, C.; MACHADO, J. M. H.; MINAYO-GOMEZ, C. Proposição de um método de análise coletiva dos acidentes de trabalho no hospital. Cadernos de Saúde Pública, Rio de Janeiro, v. 21, n. 2, p. 517-524, abr. 2005.

PANAGAKOS, F. S.; SILVERSTEIN, J. Incidence of percutaneous injuries at a dental school: a 4-year retrospective study. American Journal of Infection Control, United States, v. 25, n. 4, p.330-334, Aug.1997.

RAMOS-GOMEZ, F. et al. Accidental exposures to blood and body fluids among health care workers in dental teaching clinics: a prospective study. Journal of the American Dental Association, United States, v. 128, n. 9, p. 1253-1261, Apr. 1997.

RIBEIRO, P. H. V. Acidentes com material biológico potencialmente contaminado em alunos de um curso de odontologia do interior do estado do Paraná. 2005 112 f. Dissertação (Mestrado)-Universidade de São Paulo, Escola de Enfermagem de Ribeirão Preto, Ribeirão Preto, 2005.

SCHECHTER, M.; MARANGONI, D. V. Doenças infecciosas: conduta, diagnóstico e terapêutica. 2. ed. Rio de Janeiro: Guanabara Koogan, 1998.

SENNA, M. I. B.; GUIMARÃES, M. D. C.; PORDEUS, I. A. Atendimento odontológico de portadores de HIV/AIDS: fatores associados à disposição de cirurgiões-dentistas do Sistema Único de Saúde de Belo Horizonte, Minas Gerais, Brasil. Cadernos de Saúde Pública, Rio de Janeiro, v. 21, n. 1, p. 217-225, fev. 2005.

SILVA, P. E. B.; PATROCÍNIO, M. C.; NEVES, A. C. C. Avaliação da conduta de biossegurança em clínicas odontológicas de graduação. Revista Biociências, Taubaté, v. 8, n. 1, jan./jun. 2002.

TIPPLE, A. F. V. et al. O ensino do controle de infecção: um ensaio teórico-prático. Revista Latino-Americana de Enfermagem, Ribeirão Preto, v. 11, n. 2, p. 245-250, mar./abr., 2003.

VAN GEMERT-PIJNEN, J. et al. Effectiveness of protocols for preventing occupational exposure to blood and body fluids in dutch hospitals. Journal of Hospital Infection, England, v. 62, n. 2, 166-173, Feb. 2006.

YOUNAI, F. S.; MURPHY, D. C.; KOTELCHUCK, D. Occupational exposures to blood in a dental teaching environment: results of a ten-year surveillance study. Journal of Dental Education, United States, v. 65, n. 5, p. 436-448, May 2001. 